\title{
Laparoscopy Interfacing with MEDNET Software
}

\author{
G. M. Ghormode ${ }^{1}$, V. R. Bora ${ }^{2}$ \\ ${ }^{1}$ Student, M.Tech (Communication Engineering) Electronics and Telecommunication Engineering G. H. Raisoni College of Engineering \\ Nagpur, India \\ ${ }^{2}$ Professor, Electronics and Telecommunication Engineering G. H. Raisoni College of Engineering Nagpur, India
}

\begin{abstract}
Laparoscopy is a technique that is used for diagnosis and treatment of internal organs in abdomen by inserting a camera through incision. In this paper we are introducing a novel technique has been proposed through which we enable a Doctor to access data remotely with remote server. Here the images and video are taken by the laparoscopy camera and are then transferred to the Raspberry-Pi controller through optical fiber cable without disturbing existing Laparoscopy system. The controller then processes the data and may transmit it to the remote location server to other doctors that can help in treatment and diagnosis.
\end{abstract}

Keywords: Laparoscopy, camera, Raspberry-Pi controller, Optical fiber cable

\section{Introduction}

Laparoscopic surgery is one of the most frequent procedures performed today. It is a modern surgical technique in which operations in the abdomen are performed through small incisions. A laparoscopy is generally recommended to examineappendix, liver, small and large bowel, an abdominal mass or tumor, liver diseases, gallbladder or pancreas. This is generally done when other non-invasive methods such as Ultrasound, CT scan, and MRI does not give sufficient information for diagnosis. A patient with laparoscopic surgery has following advantages as compared a patient with an open surgery:

- Smaller cuts in the skin for surgery

- Reduced pain

- Less chances of infections

- Reduced bleeding

- Shorter recovery time

- Less hospital staying time

However, Minimally Invasive Surgery (MIS) has been significantly improved over the last few decades due the development of specialized electronic tools. Some of the features which have been improved include:

- Simulators: Use of simulators has significantly improved the proficiency of surgeons.

- Stabilization: Instability in the process may occur due to machinery or shaky human hands. This has been overcome by using stabilized machines.

- Visual magnification: Large screens and good quality cameras have improved the visibility.[2][3]

\section{Literature Survey}

Now a days, due to accidents injury in joints is common. For the treatment of these joint injuries endoscopy has played a very important part. Endoscopy is a technique similar to laparoscopy in which a small camera is inserted into the joints through a small incision for the treatment of joints. Actually endoscopy can be used for both the diagnosis and treatment. In [1] the research was focused on designing a GUI for endoscopy system that includes suggestion of doctors and can be convenient for users. Here, the authors performed a demonstrative test and have done research analysis on simulation for the improvement in the performance of endoscopy.

\section{Theory of Techniques}

\section{The light source}

A light source is used in laparoscopy to illuminate the area under operation. A good quality light source is required to obtain a good quality of image. A light source typically consists of a lamp, lenses for concentrating, a heat filer and a an intensity control circuit.

Bulb

A bulb is a very important part in the light source. Light quality depends on the type of the type of bulb used. Various types of bulbs are available in the market, some of which include: Halogen bulbs, Xenon bulbs, Incandescent bulbs and Metal halide vapor arc bulb.

\section{Halogen bulbs}

Halogen bulbs use Halogen gas that burns them with more intensity without decreasing their life. It provides a highly efficient light with excellent colors. These type lamps are cheap and may be used for laparoscopic surgery if low budget setup is required.

\section{Xenon lamps}

Xenon lamps had a spherical or elliptical envelope that is made by using quartz glass. A quartz glass can withstand high pressure and has high thermal stability. Clear, fused silica quartz is used to obtain ultimate image quality of videos. To absorb the harmful UV radiations generated during operation the silica quartz is typically doped. Xenon lamps when compared to Halogen lamps provide more natural light.

\section{Metal halide vapor arc lamp.}

In metal halide lamps a mixture of compounds is selected to produce a White light. Two types of Metal halide lamps generally used: Iron Iodide Lamp and Gallium Iodide Lamp. [4] 


\section{International Journal of Science and Research (IJSR) \\ ISSN (Online): 2319-7064}

Index Copernicus Value (2015): 78.96 | Impact Factor (2015): 6.391

\section{Laparoscope}

The word Laparoscope is derived from the two words "lapara" means flank or loin and "Skopein" means "to view or examine". Laparoscope is an instrument that is used to examine different structures inside the Abdomen or Pelvis.A variety of laparoscopes in diameter and tips are available,but the type of laparoscope that is to be used depends on the type of application. For example, for looking around corners angled tip laparoscopes may be used and for better image quality and visualization scopes with larger diameter may be used. Now, due to newer development in the technologies Virtual Reality (VR) and Augmented Reality (AR) systems are also developed. A Virtual Reality system depends only on the images generated by the computer. While in Augmented Reality system X-ray of patient or CT are used to create an enhanced $3 \mathrm{D}$ image. [5]

\section{A. Raspberry Pi Board}

A Raspberry Pi is controller that has the capability to work like a CPU of computer. Similar to a desktop es CPU we may load an operating system in it. It also has USB ports, HDMI port to connect it with the monitor, memory card slot to store the OS and other programs or data. The main signal processing unit in Raspberry Pi system is Broadcom 2835 $700 \mathrm{MHz}$ Chip designed with Advanced RISC architecture. [6]

\section{B. Raspbeery Pi Camera Module}

An ultra small and lightweight HD camera module with low cross-talk, low noise image and high sensitivity compatible with the Raspberry Pi model is shown in figure. The camera is attached with the board via. a 15-way ribbon cable. [7]

\section{Methodology}

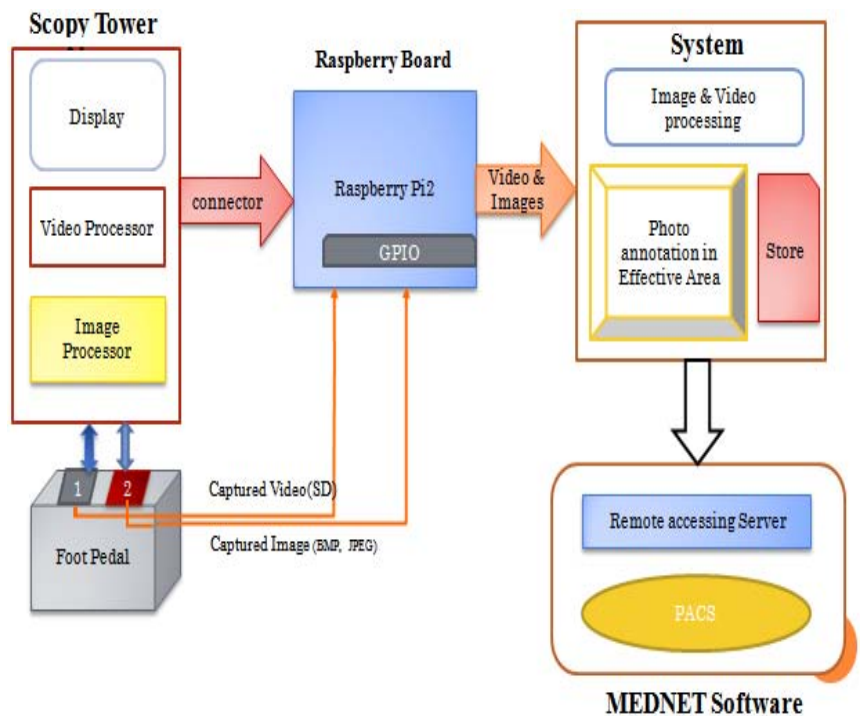

Figure: Block diagram of Laparoscopy

Fig. shows the block diagram of proposed laparoscopy based system. As shown in the diagram it consists of following main components: a light source, a digital camera (recording device), controller circuitry (controlling device), monitor (display device) and a scope (lens system).
The light source used usually is an incandescent bulb. The main disadvantage of these bulbs is the heat generated due to Infra Red (IR) radiations. This heat may be controlled by adjusting the light intensity or by using a heat absorption filter.

A Laparoscope is an instrument that consists of a number of lenses that focuses the light down in Optical fiber or gel cable. This technique is same as that used in modern digital cameras. The image quality highly depends on the design and the type of lens used.The lenses are used to convert the light source into a single narrow beam by focusing them on a single point. This beam is then transmitted to the Laparoscope via. Either an optical fiber cable or a gel cable. These cables are usually used because they have a high transmission levels but are little delicate. As compare to fiber optic cables, gel cables are superior in brightness and color temperature but are more fragile. A Laparoscope also consists of rod lenses through which internal pictures of the body taken by camera are transmitted. A number of Laparoscopes are available in the market depending on the number of rods, diameter of lenses, its overall length and the viewing angle but a wider scope gives brighter images.

The images taken by the Laparoscope or camera are analog in nature so these images are then converted into digital form. Once these images are digitized these are processed by digital image processing techniques to improve the quality of image by reducing noise, adjusting colors and enhancement. These resulting images may then be applied to the highdefinition monitors through a remote accessing server by the MEDNET software so that other surgeons can also monitor.

\section{Flowchart}

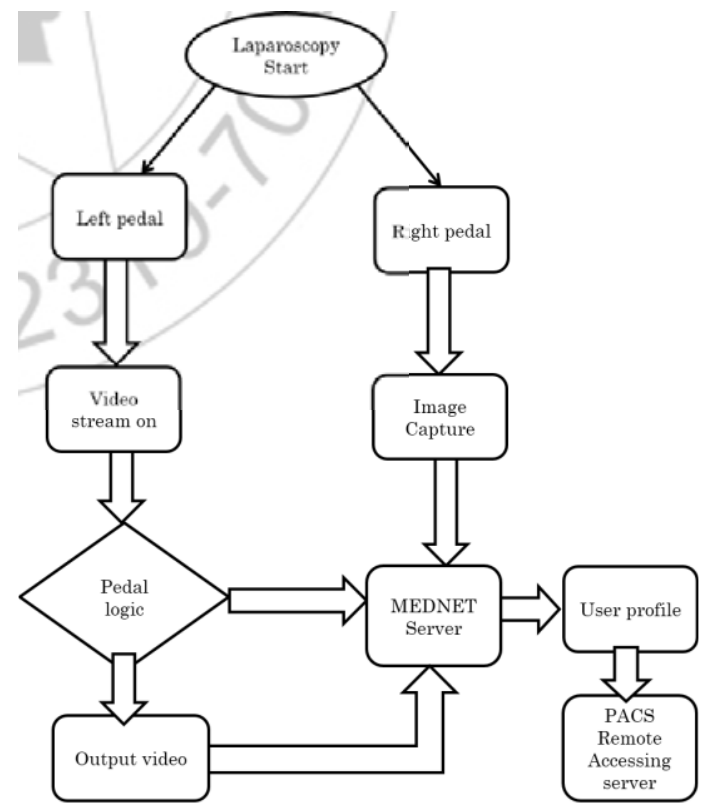

\section{Conclusion}

Variouslaparoscopic methods are proposed in different papers.We reviewed these papers and proposed our noninvasive method of Laparoscopy Integration Over The traditional method of laparoscopy. 


\section{International Journal of Science and Research (IJSR) \\ ISSN (Online): 2319-7064}

Index Copernicus Value (2015): 78.96 | Impact Factor (2015): 6.391

\section{References}

[1] Jong An Park, Minhhyuk Chang „Development of a Lowcost PC-based Endoscope System ${ }^{e}$ (ICHIT'06) 2006 IEEE.

[2] http://www.healthline.com/health/laparoscopy\#Purpose2

[3] http://www.healthline.com/health/laparoscopy\#Overview 1

[4] https://www.laparoscopyhospital.com/PR01.HTM\#Xeno $\mathrm{n} \%$ 20lamps.

[5] http://www.laparoscopic.md/instruments/laparoscope

[6] Sahil R. Arora1, Prof. Amol Boke2, Prof. Pragati Kene2, Suraj U. Patinge3 “Advanced Real Time Home Security System Based On Raspberry Pi” International Journal Of Pure And Applied Research In Engineering And Technology

[7] M. Roelands et ai., "Enabling the masses for become creative and smart space", in Architecting the Internet of Things, Berlin, Germany Springer-Verlag, 2011, pp 3843. 